\title{
Hypoxia at the heart of sudden infant death syndrome?
}

\author{
Marianne T. Neary' and Ross A. Breckenridge ${ }^{1,2}$
}

Sudden infant death syndrome (SIDS) is a significant clinical problem without an accepted pathological mechanism, but with multiple conflicting models. Mutations in a growing number of genes have been found postmortem in SIDS cases, notably genes encoding ion channels. This can only account for a minority of cases, however. Our recent work on a novel mouse model of SIDS suggests a potentially more widespread role for cardiac arrhythmia in SIDS without needing to invoke the inheritance of abnormal ion-channel genes. We propose a model for SIDS pathogenesis whereby postnatal hypoxia leads to delayed maturation of the cardiac conduction system and an increased risk of cardiac arrhythmia. Our model may integrate several epidemiological findings related to risks factors for SIDS, and agrees with previous work suggesting a common final pathological pathway in SIDS.

udden infant death syndrome (SIDS, also known as "cot $\checkmark$ death") remains an intractable clinical problem. No definitive unifying disease mechanism has been described, and there are several competing models of pathogenesis, all with some circumstantial supporting evidence. The definition of SIDS is an unexplained, sudden death of an infant below $1 \mathrm{y}$ of age (1). A diagnosis of SIDS can be made only after forensic examination of the death scene and postmortem pathological examination that includes a range of additional investigations (1). Incidence in the United Kingdom has been estimated at 0.40 deaths per live birth (2).

Our poor understanding of SIDS as a syndrome is a result of the fact that this is a diagnosis of exclusion, and the likelihood is that a number of different causative pathologies are lumped together as "SIDS" (3). There is also the general difficulty in ascertaining a definitive cause of death purely from postmortem pathology. However, there is a well-documented common set of postmortem pathological findings in the majority of SIDS cases, as recently reviewed by Goldwater (3). This has led to the view that a wide number of pathologies result in a final common pathway and sudden death.

The absence of a clear single pathological mechanism in SIDS led to the development of a "multi-hit" hypothesis, whereby the combination of several risk factors (which may be insignificant on their own) leads to death. The "triple risk hypothesis" was advanced in 1994 and centered on abnormal brainstem function $(4,5)$. Alterations in brain parenchymal structure and neurotransmitter release have also been described in the periaqueductal gray matter (6), arcuate nucleus (7), medulla oblongata (8), and carotid body (9). Whether these lesions are causative or merely reflect chronic hypoventilation/hypoxia remains to be determined. A retrospective case review found evidence of an unexpectedly high incidence of Staphylococcus aureus and Escherichia coli colonization/infection in SIDS cases (10), supporting the hypothesis that bacterial infection is involved in SIDS pathogenesis $(11,12)$. Furthermore, it has also been shown that cigarette smoke exposure, a known SIDS risk factor, attenuates the immune response to bacterial infection, and so might augment the effect of bacterial infection (13). Sleeping in the prone position, another risk factor for SIDS, may activate temperature-sensitive bacterial toxins (14), emphasizing the complexity of the interactions of multiple etiological factors in SIDS. These interactions are incompletely understood. For example, in an Austrian epidemiological study, incidence of SIDS increased with altitude, but this was only in prone sleepers (15). Interestingly, the study of whether sleeping in the prone position per se lowers oxygen levels has produced equivocal results (16). More recently, evidence has been advanced to support the role of catastrophic diaphragmatic weakness as a causative factor in SIDS (17). How this relates to established epidemiological risk factors remains to be seen. More widespread and efficient use of genome sequencing is suggesting other inherited risk factors, e.g., mutant sarcomere protein genes (18), and in future this technology will provide more candidate genes and perhaps mechanistic insight.

Several autopsy findings are commonly reported in victims of SIDS, although there is considerable heterogeneity, consistent with multiple causative etiologies for SIDS. Intrathoracic petechiae are regularly reported (19), as is dilated right ventricle and hepatic erythropoiesis, consistent with chronic hypoventilation $(3,20)$. More recently, anatomical abnormalities in the cardiac conduction system and autonomic nervous system have been reported in SIDS victims (21-24). In addition, it was reported in one series that hearts in SIDS cases were significantly smaller than controls, implying that postnatal growth and maturation of the heart is somehow affected in SIDS victims (25).

It is possible that cardiac arrhythmia is the ultimate mode of death in SIDS, as is the case in many adult cases of sudden death. Several pieces of circumstantial evidence support a role 


\section{Integrated Mechanism Review}

for cardiac arrhythmia in SIDS. A large prospective study of 33,034 infants found that $50 \%$ of the infants who died of SIDS had a prolonged QTc interval in the first week of life (26). In general pediatric and adult populations, QTc prolongation has long been accepted as a marker for increased susceptibility to ventricular arrhythmia $(27,28)$, and inherited channelopathies with prolonged QTc are associated with sudden death in both pediatric and adult populations (29). "Molecular autopsy" of 173 cases of SIDS, by genotyping for five genes associated with long QT syndrome and the ryanodine receptor 2 gene, revealed mutations in these arrhythmia-sensitizing genes in 44 out of 173 of cases (26\%) (30). Although this is an increase on the $\sim 10 \%$ of SIDS cases previously thought to carry functionally significant genetic variants in sodium and potassium channels causing prolonged QT duration (31), the majority of cases have no obvious molecular substrate at the moment.

Further circumstantial evidence linking SIDS to changes in QTc comes from the study of electrocardiography changes after birth. It is perhaps under-appreciated that the neonatal human cardiac conduction system remodels following birth, with increasing QTc interval up to 4 postnatal months, then significant decreases in QTc and PR interval throughout childhood to adolescence $(32,33)$. It is well known that sensitivity to SIDS parallels this change: decreasing after 4 mo of birth, when QTc is known to peak in humans (33).

Postnatal remodeling of the cardiac conduction system is incompletely understood, and is one area where animal studies are required. Efforts are currently underway to analyze postnatal cardiac conduction system remodeling in mice, for example by using mouse lines expressing green fluorescent protein in conduction tissue (34). This process is thought to involve a controlled program of apoptosis and proliferation called "resorptive degeneration" (35). A full description is out of the scope of this review-the interested reader is directed to a review by Ottaviani et al. (35). Anatomical abnormalities of the cardiac conduction system related to abnormal postnatal maturation are likely to be under-recognized in SIDS victims, probably as a result of under-investigation at autopsy (36). Abnormal conduction tissue, such as accessory pathways and abnormal nodal architecture (37), and evidence of defective remodeling (38) have been reported in autopsies of SIDS victims, which could very plausibly lead to fatal cardiac arrhythmia (as it does in the fetus), although the contribution of this to the overall incidence of SIDS cases is somewhat contentious (39). Furthermore, epidemiological studies have linked maternal smoking and, specifically, exposure to smoking-derived carbon monoxide (recognized risk factors for SIDS) with histological abnormality in the cardiac conduction system (40). The generally poor adherence to standardized protocols for postmortem examination of SIDS victims $(41,42)$ has probably led to under-recognition of the role of abnormal postnatal cardiac conduction system development in the pathogenesis of SIDS. It has been argued that formal histological examination of the central nervous system and cardiac conduction system of SIDS victims is warranted, despite the labor-intensive nature of such studies, as it will

\section{Neary et al.}

give a clearer picture of the prevalence of abnormalities in these tissues (43).

In addition to the circumstantial evidence linking QTc to SIDS, most risk factors for SIDS are associated with a reduced oxygen environment. Multiple risk factors for SIDS have been discovered from epidemiological studies of SIDS victims, including prone sleeping position (44), head covering, respiratory control disorders (45), maternal smoking (46), and high altitude $(15,47,48)$. The result of epidemiological work has been arguably the major advance in prevention of SIDS. The "back to sleep" campaign, which started in the 1990s, encouraged parents to only allow their babies to sleep on their backs. It has been estimated that this led to a sustained 50\% drop in the SIDS incidence rate, which has gradually declined further since then. These environmental risk factors for SIDS could all lead to hypoventilation and relative hypoxia. Furthermore, interventions that increase ventilation in an infant's room show reductions in SIDS incidence; a study looking at fan use suggested a $72 \%$ reduction in SIDS incidence when a fan was used to ensure better air circulation in the room (49). Hypoxia is implicated in risks of an organic nature too: immaturity of the respiratory centers in the brainstem might cause fatal hypoventilation, known as "congenital central hypoventilation syndrome" $(4,5,45)$, and diaphragmatic malfunction may lead to exacerbation of hypoventilation and thus fatal hypoxia (17).

We hypothesize that neonatal hypoxia leads to abnormal electrical conduction as a potential cause of, or contribution to, sudden death in neonates. There are established links among hypoxia, cardiac ischemia, prolonged QTc, and ventricular arrhythmia in adults $(50,51)$. Human populations at high altitude have been found to exhibit a high SIDS rate (47) and higher rates of unexplained neonatal death in hospital (52). Interestingly, QTc has been found to be relatively prolonged in high-altitude pediatric populations compared with lower altitude controls (53). We therefore studied the effect of hypoxia and elevated hypoxia signaling on neonatal mice to investigate the role of ambient oxygen levels in neonatal cardiac maturation and sensitivity to sudden death (54).

We found that maturation of the cardiac conduction system after birth in mice is dependent on reduced levels of hypoxia signaling in the myocardium. Mice, like humans, undergo a postnatal process of cardiac electrophysiological maturation, as evidenced by progressive decreases in QTc duration over the first postnatal month. Mice either born into a hypoxic environment or genetically engineered to have increased myocardial hypoxia signaling exhibit delayed electrocardiac maturation and greatly increased rates of sudden death (54). These experimental conditions also prevented the usual postnatal changes in expression of genes encoding ion channels, some of which have been implicated in human SIDS cases. Significantly, we found that risk of death of wild-type neonatal mice decreased with increasing time between birth and exposure to hypoxia, defining a period of sensitivity to hypoxia-induced sudden cardiac death. We therefore hypothesize that neonatal hypoxia leads to cardiac gene expression changes that increase the risk of arrhythmogenic death. 


\section{Hypoxia and sudden infant death syndrome}

We have not observed any changes in chamber architecture from mice sacrificed shortly before the sudden death of their siblings, nor are aMHC-Cre:: $\mathrm{VHL}^{(\mathrm{f} / \mathrm{fl})}$ hearts smaller than their nontransgenic siblings (our unreported data). This suggests that the changes in cardiac chamber architecture in SIDS may be due to extracardiac factors such as pulmonary remodeling or hypoventilation. aMHC-Cre:: $\mathrm{VHL}^{(\mathrm{f} / \mathrm{fl})}$ mice have genetic modification limited to the myocardium and exhibited the same respiratory rate as their nontransgenic siblings (unpublished data). Furthermore, we have not observed petechiae on the cardiac surface of dead pups (although studies are continuing). However, postmortem examination of neonatal mice is hampered by extremely rapid degeneration of tissue and immediate eating of dead pups by their mother, emphasizing one significant limitation of the murine system. Examination of changes in morphology of the cardiac conduction system was not carried out in our initial characterization of the aMHC-Cre::VHL ${ }^{(\mathrm{f} / \mathrm{fl})}$ model. These studies are ongoing and may strengthen our hypothesis placing postnatal hypoxia at the center of SIDS pathogenesis. We believe that this mouse model will prove useful in studying the molecular and metabolic changes leading up to sudden cardiac death.

Currently, mutations in ion-channel genes can only explain a small proportion of SIDS cases. Our hypoxia-arrhythmia model would suggest that ambient oxygen levels alter ion-channel expression levels (i.e., alteration in expression level of a wild-type gene product rather than sequence mutation, meaning that gene sequencing will be unhelpful). Our finding that connexin 43 (Cx43) phosphorylation decreased in hypoxic neonatal mouse hearts provides another potential mechanism of arrhythmia. Indeed, mutations in $\mathrm{Cx} 43$ have been reported postmortem in some SIDS cases (55), and dephosphorylation of $\mathrm{Cx} 43$ has been found to lead to electrical uncoupling during cardiac ischemia in adult hearts, which may lead to arrhythmia (56).

We therefore propose a model of SIDS pathogenesis whereby hypoxia (resulting from several causes) during a critical postnatal period leads to delayed maturation of the cardiac conduction system and increased probability of cardiac arrhythmia (Figure 1). We suggest that our model is unlikely to be

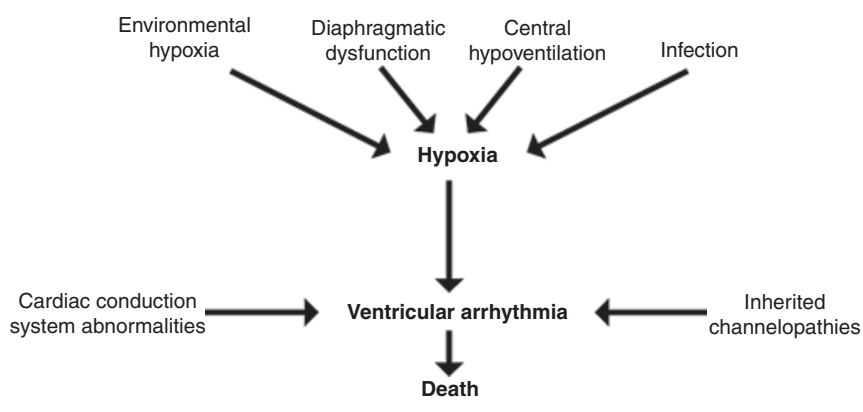

Figure 1. Suggested model for the pathogenesis of sudden infant death syndrome (SIDS). Myocardial hypoxia, from multiple possible causes, leads to an increased risk of ventricular arrhythmia. The broad term "cardiac conduction system abnormalities" covers conduction system defects resulting from generalized cardiac developmental abnormalities and those resulting from abnormal postnatal maturation of the cardiac conduction system.

\section{Integrated Mechanism Review}

exclusive of the other risk factors and pathological mechanisms briefly mentioned here; it is likely that defects in the central nervous system, carotid body, and diaphragm lead to hypoxia, and thus afford an additional risk of death in combination with environmental factors such as sleeping position. Given findings of abnormal cardiac conduction system anatomy and histopathology $(35,38,57)$, it is an interesting question whether the postnatal development of the cardiac conduction system, by processes such as resorptive degeneration, is dependent on adequate oxygen levels.

In the face of a multiplicity of contributing pathologies while the majority of cases exhibit the same postmortem pathological findings, our model suggests a final common pathway of cardiac arrhythmia for SIDS, with several potential entrance routes. One challenging finding is the apparent relatively low incidence of human SIDS in the first postnatal month (58). In theory, this could be due to the fact that the mammalian fetus is adapted to profoundly low absolute levels of oxygen in utero and that this adaptation persists during the first month after birth. The mechanisms of fetal hypoxia protection remain to be defined.

A maturational effect of hypoxia on the myocardium following birth also has implications for cyanotic congenital heart disease. It is currently unknown whether congenital cardiac malformations leading to cyanosis lead to the same sort of electrocardiographic "immaturity" as mice reared in hypoxic conditions following birth. If this is the case, an obvious question is whether timing of surgical correction might influence eventual incidence of cardiac arrhythmia. Certainly, patients with repaired congenital cardiac lesions exhibit high rates of ventricular arrhythmia and sudden death (59), but there are many confounding factors, such as the influence of myocardial surgical scars and abnormal embryonic development of the conduction system. This question might be addressed by animal modeling and novel imaging techniques in human patients, such as those using cardiac magnetic resonance imaging/nuclear magnetic resonance.

There are some important gaps in our understanding of the maturation of the mammal in the immediate period following birth. In addition to the maturation of cardiac electrical conduction, the cardiovascular system undergoes rapid and profound morphological remodeling in the immediate period following birth. Maturation of the brainstem, and in particular the pontine respiratory centers, occurs after birth and is little understood $(60,61)$. It is therefore possible that the infant is vulnerable to hypoxia during the first year of postnatal life for a variety of reasons, as yet unrecognized.

\section{THE FUTURE}

As whole-genome sequencing becomes cheaper and more practical, it is very likely that new genetic mutations associated with SIDS will be identified. This may allow dissection of the complex web of pathological findings associated with SIDS. Certainly, advances in the understanding of maturational processes in the heart, brainstem, immune system, and diaphragm following birth will be helpful. 
As regards the specific hypothesis that hypoxia leads to delayed myocardial maturation and vulnerability to arrhythmia, animal modeling of SIDS and the search for novel biomarkers of hypoxic stress in neonatal mouse and human hearts are already underway. We agree that in the context of a serious medical problem with no clear pathological basis, animal models of SIDS are a vital and arguably under-used tool (3). Ultimately, however, there seems to be no substitute for large, prospective epidemiological studies combined with advanced phenotyping and in-depth necropsy studies concentrating on detailed histological examination of the cardiac conduction system and autonomic nervous system.

In summary, we suggest hypoxia could be common mechanism for a large proportion of SIDS cases via hypoxia-mediated changes in gap junction electrical coupling, ion-channel expression and myocardial maturation. Currently $\sim 1$ in 2,000 infants are victims to SIDS, and this rate remains stubbornly high, despite the continuing success of the "back to sleep" campaign in started in $1994(62,63)$. A common mechanism transforms a seemingly disparate series of advice to parents into one simple message around avoiding a low-oxygen environment and sets in motion more obvious preventative measures. These could range from a simple fan and an open door or window to an oxygen tent for high-altitude infants. Electrocardiography screening of infants on attendance of routine health checks and vaccinations would help to identify those infants who are particularly susceptible owing to prolonged QTc intervals and for whom hypoxia-reducing interventions are the most important. Finally, we believe that in the face of a serious condition with multiple competing theories of etiology, animal modeling will be vital for further reductions in mortality.

\section{STATEMENT OF FINANCIAL SUPPORT}

No financial assistance was received to support this study.

\section{REFERENCES}

1. Royal College of Pathologists. Sudden unexplained death in infancy. A multi-agency protocol for care and investigation, 2004. (http://www. rcpath.org/NR/rdonlyres/30213EB6-451B-4830-A7FD-4EEFF0420260/0/ SUDIreportforweb.pdf).

2. UK National Office for Statistics. Unexplained deaths in infancy, 2009. (http://www.ons.gov.uk/ons/rel/child-health/unexplained-deaths-ininfancy-england-and-wales/2009/new-component.html).

3. Goldwater PN. A perspective on SIDS pathogenesis. the hypotheses: plausibility and evidence. BMC Med 2011;9:64.

4. Filiano JJ, Kinney HC. A perspective on neuropathologic findings in victims of the sudden infant death syndrome: the triple-risk model. Biol Neonate 1994;65:194-7.

5. Guntheroth WG, Spiers PS. The triple risk hypotheses in sudden infant death syndrome. Pediatrics 2002;110:e64.

6. Sawaguchi T, Franco P, Kato I, et al. Interaction between apnea, prone sleep position and gliosis in the brainstems of victims of SIDS. Forensic Sci Int 2002;130:Suppl:S44-52.

7. Matturri L, Biondo B, Mercurio P, Rossi L. Severe hypoplasia of medullary arcuate nucleus: quantitative analysis in sudden infant death syndrome. Acta Neuropathol 2000;99:371-5.

8. Obonai T, Asanuma M, Mizuta R, Horie H, Tanaka J, Takashima S. Evidence of brain ischemia in early neonatal sudden death syndrome. Neuropediatrics 1997;28:145-8.

9. Naeye RL, Fisher R, Ryser M, Whalen P. Carotid body in the sudden infant death syndrome. Science 1976;191:567-9.
Neary et al.

10. Weber MA, Klein NJ, Hartley JC, Lock PE, Malone M, Sebire NJ. Infection and sudden unexpected death in infancy: a systematic retrospective case review. Lancet 2008;371:1848-53.

11. Morris JA. The common bacterial toxins hypothesis of sudden infant death syndrome. FEMS Immunol Med Microbiol 1999;25:11-7.

12. Morris JA, Haran D, Smith A. Hypothesis: common bacterial toxins are a possible cause of the sudden infant death syndrome. Med Hypotheses 1987;22:211-22.

13. Raza MW, Essery SD, Elton RA, Weir DM, Busuttil A, Blackwell C. Exposure to cigarette smoke, a major risk factor for sudden infant death syndrome: effects of cigarette smoke on inflammatory responses to viral infection and bacterial toxins. FEMS Immunol Med Microbiol 1999;25:145-54.

14. Blackwell CC, Moscovis SM, Gordon AE, et al. Cytokine responses and sudden infant death syndrome: genetic, developmental, and environmental risk factors. J Leukoc Biol 2005;78:1242-54.

15. Kohlendorfer U, Kiechl S, Sperl W. Living at high altitude and risk of sudden infant death syndrome. Arch Dis Child 1998;79:506-9.

16. Peirano P, Guidasci S, Monod N. Effect of sleep position on transcutaneous oxygen tension in SIDS siblings. Early Hum Dev 1986;13:303-12.

17. Siren PM, Siren MJ. Critical diaphragm failure in sudden infant death syndrome. Ups J Med Sci 2011;116:115-23.

18. Brion M, Allegue C, Santori M, et al. Sarcomeric gene mutations in sudden infant death syndrome (SIDS). Forensic Sci Int 2012;219:278-81.

19. Krous HF, Haas EA, Chadwick AE, Masoumi H, Stanley C. Intrathoracic petechiae in SIDS: a retrospective population-based 15-year study. Forensic Sci Med Pathol 2008;4:234-9.

20. Naeye RL, Whalen P, Ryser M, Fisher R. Cardiac and other abnormalities in the sudden infant death syndrome. Am J Pathol 1976;82:1-8.

21. Lavezzi AM, Matturri L. Neuroanatomical dysmorphology of the medial superior olivary nucleus in sudden fetal and infant death. Front Hum Neurosci 2012;6:322.

22. Lavezzi AM, Weese-Mayer DE, Yu MY, et al. Developmental alterations of the respiratory human retrotrapezoid nucleus in sudden unexplained fetal and infant death. Auton Neurosci 2012;170:12-9.

23. Matturri L, Lavezzi AM, Minoli I, et al. Association between pulmonary hypoplasia and hypoplasia of arcuate nucleus in stillbirth. J Perinatol 2003;23:328-32.

24. Ottaviani G, Lavezzi AM, Matturri L. Fibromuscular hyperplasia of the pulmonary artery in sudden infant and perinatal unexpected death. Cardiovasc Pathol 2009;18:223-30.

25. Kelmanson IA. Retarded heart growth in the victims of sudden infant death: possible implications for lethal mechanisms. Eur J Pediatr 1996;155:440-4.

26. Schwartz PJ, Stramba-Badiale M, Segantini A, et al. Prolongation of the QT interval and the sudden infant death syndrome. N Engl J Med 1998;338:1709-14.

27. Gale GE, Bosman CK, Tucker RB, Barlow JB. Hereditary prolongation of QT interval. Study of two families. Br Heart J 1970;32:505-9.

28. Bokil NJ, Baisden JM, Radford DJ, Summers KM. Molecular genetics of long QT syndrome. Mol Genet Metab 2010;101:1-8.

29. Skinner JR, Crawford J, Smith W, et al.; Cardiac Inherited Disease Group New Zealand. Prospective, population-based long QT molecular autopsy study of postmortem negative sudden death in 1 to 40 year olds. Heart Rhythm 2011;8:412-9.

30. Tester DJ, Medeiros-Domingo A, Will ML, Haglund CM, Ackerman MJ. Cardiac channel molecular autopsy: insights from 173 consecutive cases of autopsy-negative sudden unexplained death referred for postmortem genetic testing. Mayo Clin Proc 2012;87:524-39.

31. Arnestad M, Crotti L, Rognum TO, et al. Prevalence of long-QT syndrome gene variants in sudden infant death syndrome. Circulation 2007;115:3617.

32. Rautaharju PM, Davignon A, Soumis F, Boiselle E, Choquette A. Evolution of QRS-T relationship from birth to adolescence in Frank-lead orthogonal electrocardiograms of 1492 normal children. Circulation 1979;60:196-204.

33. Schaffer MS, Trippel DL, Buckles DS, Young RH, Dolan PL, Gillette PC. The longitudinal time course of QTc in early infancy. Preliminary results of a prospective sudden infant death syndrome surveillance program. J Perinatol 1991;11:57-62. 


\section{Hypoxia and sudden infant death syndrome}

34. Miquerol L, Moreno-Rascon N, Beyer S, et al. Biphasic development of the mammalian ventricular conduction system. Circ Res 2010;107:153-61.

35. Ottaviani G, Matturri L, Rossi L, James TN. Crib death: further support for the concept of fatal cardiac electrical instability as the final common pathway. Int J Cardiol 2003;92:17-26.

36. James TN. Normal variations and pathologic changes in structure of the cardiac conduction system and their functional significance. J Am Coll Cardiol 1985;5:Suppl 6:71B-8B.

37. Matturri L, Ottaviani G, Ramos SG, Rossi L. Sudden Infant Death Syndrome (SIDS): a study of cardiac conduction system. Cardiovasc Pathol 2000;9:137-45.

38. Matturri L, Ottaviani G, Lavezzi AM, Turconi P, Cazzullo A, Rossi L. Expression of apoptosis and proliferating cell nuclear antigen (PCNA) in the cardiac conduction system of crib death (SIDS). Adv Clin Path 2001;5:79-86.

39. Anderson RH. Sudden and unexpected death in infancy and the conduction system of the heart. Cardiovasc Pathol 2000;9:147-8.

40. Sartiani L, Stillitano F, Luceri C, et al. Prenatal exposure to carbon monoxide delays postnatal cardiac maturation. Lab Invest 2010;90: 1582-93.

41. Matturri L, Ottaviani G, Lavezzi AM. Techniques and criteria in pathologic and forensic-medical diagnostics in sudden unexpected infant and perinatal death. Am J Clin Pathol 2005;124:259-68.

42. Matturri L, Ottaviani G, Lavezzi AM. Guidelines for neuropathologic diagnostics of perinatal unexpected loss and sudden infant death syndrome (SIDS): a technical protocol. Virchows Arch 2008;452:19-25.

43. Ottaviani G. Sudden infant and perinatal unexplained death: are we moving forward yet? Cardiovasc Pathol 2011;20:302-6.

44. Schnitzer PG, Covington TM, Dykstra HK. Sudden unexpected infant deaths: sleep environment and circumstances. Am J Public Health 2012;102:1204-12.

45. Thach BT. The role of respiratory control disorders in SIDS. Respir Physiol Neurobiol 2005;149:343-53.

46. Blair PS, Fleming PJ, Bensley D, et al. Smoking and the sudden infant death syndrome: results from 1993-5 case-control study for confidential inquiry into stillbirths and deaths in infancy. Confidential Enquiry into Stillbirths and Deaths Regional Coordinators and Researchers. BMJ 1996;313:195-8.

47. Barkin RM, Hartley MR, Brooks JG. Influence of high altitude on sudden infant death syndrome. Pediatrics 1981;68:891-2.

48. Getts AG, Hill HF. Sudden infant death syndrome: incidence at various altitudes. Dev Med Child Neurol 1982;24:61-8.

\section{Integrated Mechanism Review}

49. Coleman-Phox K, Odouli R, Li DK. Use of a fan during sleep and the risk of sudden infant death syndrome. Arch Pediatr Adolesc Med 2008;162:963-8.

50. Roche F, Reynaud C, Pichot V, et al. Effect of acute hypoxia on QT rate dependence and corrected QT interval in healthy subjects. Am J Cardiol 2003;91:916-9.

51. Tirlapur VG, Mir MA. Nocturnal hypoxemia and associated electrocardiographic changes in patients with chronic obstructive airways disease. $\mathrm{N}$ Engl J Med 1982;306:125-30.

52. Yangzom Y, Qian L, Shan M, et al. Outcome of hospital deliveries of women living at high altitude: a study from Lhasa in Tibet. Acta Paediatr 2008;97:317-21.

53. Fuenmayor AJ, Stock FU, Fuenmayor AC, Fuenmayor P AM. QT interval and final portion of $\mathrm{T}$ wave: measurements and dispersion in infants born at high altitude. Int J Cardiol 2002;82:123-6.

54. Neary MT, Mohun TJ, Breckenridge RA. Hypoxia, long QT interval and sudden infant death syndrome (SIDS). Dis Model Mech 2013;6:279.

55. Van Norstrand DW, Asimaki A, Rubinos C, et al. Connexin43 mutation causes heterogeneous gap junction loss and sudden infant death. Circulation 2012;125:474-81.

56. Beardslee MA, Lerner DL, Tadros PN, et al. Dephosphorylation and intracellular redistribution of ventricular connexin 43 during electrical uncoupling induced by ischemia. Circ Res 2000;87:656-62.

57. Ottaviani G, Matturri L, Rossi L, Jones D. Sudden death due to lymphomatous infiltration of the cardiac conduction system. Cardiovasc Pathol 2003;12:77-81.

58. Sullivan FM, Barlow SM. Review of risk factors for sudden infant death syndrome. Paediatr Perinat Epidemiol 2001;15:144-200.

59. Koyak Z, Harris L, de Groot JR, et al. Sudden cardiac death in adult congenital heart disease. Circulation 2012;126:1944-54.

60. Putnam RW, Conrad SC, Gdovin MJ, Erlichman JS, Leiter JC. Neonatal maturation of the hypercapnic ventilatory response and central neural $\mathrm{CO}_{2}$ chemosensitivity. Respir Physiol Neurobiol 2005;149:165-79.

61. Achard P, Zanella S, Rodriguez R, Hilaire G. Perinatal maturation of the respiratory rhythm generator in mammals: from experimental results to computational simulation. Respir Physiol Neurobiol 2005;149:17-27.

62. National Institute for Child Health and Disease, 2006. SIDS Rate and Back Sleeping (1988-2006). (http://www.nichd.nih.gov/SIDS/Documents/ SIDS_rate_back_sleep_2006.pdf).

63. U.S. Centers for Disease Control and Prevention. National Vital Statistics Reports. 1998-2012; vol. 61. (http://www.cdc.gov/nchs/products/nvsr.htm). Accessed 30 May 2013. 